\title{
Asociación entre tuberculosis y diabetes mellitus en el primer nivel de atención
}

\author{
DOI: https://doi.org/10.5377/alerta.v3i1.8741
}

\section{Marbel Alexander Magaña Revelo ${ }^{{ }^{*}}$, Ingrid América Rivas Hernández ${ }^{2}$, Julio Cesar Morales Cruz ${ }^{3}$, Milton Alonso Alfaro Vásquez ${ }^{4}$}

1. Doctor en medicina, Máster en salud pública, Máster en epidemiología, Coordinador de la Unidad de Vigilancia en Salud, Dirección Regional de Salud Metropolitana, Ministerio de Salud, San Salvador, El Salvador.

2. Doctor en Medicina, Médico consultante, Unidad Comunitaria de Salud Familiar Santiago Texacuangos, Ministerio de Salud, Santiago Texacuangos, San Salvador, El Salvador.

3. Doctor en Medicina. Colaborador técnico médico, Unidad de Gestión de Servicios de Salud, Dirección de Salud Metropolitana, Ministerio de Salud, San Salvador, El Salvador.

4. Doctor en Medicina, Coordinador de la Unidad de Abastecimiento, Dirección Regional de Salud Metropolitana, Ministerio de Salud, San Salvador, El Salvador.

${ }^{*}$ Correspondencia

Mmarbelmagana@hotmail.com

(D) 0000-0003-0595-4316.

\begin{abstract}
Resumen
Introducción. En octubre 2018 inició la estrategia de atención integral de pacientes con tuberculosis y diabetes mellitus en seis unidades comunitarias de salud familiar de la región metropolitana. Dicha estrategia brinda atención integral a pacientes con estas enfermedades. Objetivo. Establecer la asociación entre tuberculosis y diabetes mellitus en establecimientos que han implementado el plan de atención integral para tuberculosis y diabetes mellitus. Metodología. Estudio transversal analítico donde se estudiaron 12 casos nuevos con tuberculosis y diabetes mellitus, mayores de 10 años, sin otro factor de riesgo. Resultados. La prevalencia de diabetes mellitus en los casos de tuberculosis en los establecimientos donde se ha implementado el plan, es mayor que en la población general de El Salvador $(p=0.02)$. No existe diferencia significativa entre la prevalencia obtenida en los establecimientos donde se ha implementado el plan y los resultados a nivel nacional para ambos sexos (femenino 0.09 y masculino 0.07). La probabilidad de padecer tuberculosis es 11.4 veces mayor entre los diabéticos. Conclusiones. Existe una asociación significativa entre la diabetes mellitus y tuberculosis en los establecimientos donde se ha implementado el plan de atención integral para tuberculosis y diabetes mellitus. La probabilidad de enfermar de tuberculosis en los diabéticos es mayor que en los no diabéticos.
\end{abstract}

Palabras claves

Tuberculosis, diabetes mellitus, comorbilidad, grupos de riesgo, epidemiología.

\section{Abstract}

Introduction. In October 2018, the strategy of comprehensive care for patients with tuberculosis and diabetes mellitus began in 6 community family health units in the metropolitan region, a strategy that provides comprehensive care for patients with these diseases. Objective. Establish the association between tuberculosis and diabetes mellitus, in establishments that have implemented the comprehensive care plan for tuberculosis and diabetes mellitus. Methodology. This study is an analytical cross-section where 12 new cases with tuberculosis and diabetes mellitus, older than 10 years, with no other risk factor were studied. Results. The prevalence of diabetes mellitus in cases of tuberculosis in the establishments where the plan has been implemented is higher than in the general population of El Salvador $(p=0.02)$. There is no significant difference between the prevalence obtained in the establishments where the plan has been implemented and the results at the national level for both sexes (female 0.09 and male 0.07 ). The probability of suffering from tuberculosis among diabetics is 11.4. Conclusions. The association of diabetes mellitus and tuberculosis is greater in the establishments where the comprehensive care plan for tuberculosis and diabetes mellitus has been implemented in comparison to the general population. The probability of getting tuberculosis in diabetics is higher than in non-diabetics.
\end{abstract}

Keywords

Tuberculosis, diabetes mellitus, comorbidity, risk groups, epidemiology.
G ACCESO ABIERTO

Association between tuberculosis and diabetes mellitus in primary health

Citación recomendada:

Magaña Revelo MA, Rivas Hernández IA, Morales Cruz JC, Alfaro Vásquez MA. Asociación entre tuberculosis y diabetes mellitus en el primer nivel de atención. Alerta 2020; 3(1):13-17. DOI: https://doi. org/10.5377/alerta.v3i1.8741

Recibido:

15 de noviembre de 2019

\section{Aceptado:}

6 de enero de 2020

Publicado:

27 de enero de 2020

Contribución de autoría: MAMR': Concepción de estudio. MAMR', IARH². $J_{C M C^{3}}, M_{A A V}$ : Diseño de estudio, búsqueda, análisis e interpretación de datos, borrador del artículo y rendición de cuentas de todos los aspectos de la investigación.

\section{Conflicto de intereses:}

Los autores no tienen conflicto de intereses. 


\section{Introducción}

En la primera conferencia mundial ministerial de la Organización Mundial de la Salud, se plantea para el año 2030, poner fin a la tuberculosis (TB) a través de compromisos como el de lograr sinergias en la gestión de la TB, coinfecciones y enfermedades no transmisibles'.

En El Salvador, durante el 2016 se reportó un total de 3034 casos nuevos de TB en todas las formas ${ }^{2}$ y durante los últimos 5 años se ha tenido un aumento del 10\% de esta enfermedad. Entre los grupos de riesgo identificados de padecer TB están las personas privadas de libertad, quienes representaron el 32\% de los casos en el año 20162; y el $6.6 \%$ de los casos de coinfección TB/VIH².

Las personas con diabetes mellitus son susceptibles al aparecimiento de la TB. En el 2016 se reportaron 13192 casos nuevos de diabetes mellitus $(\mathrm{DM})^{3}$, sin embargo, se conoce poco sobre la comorbilidad TB-DM en el país. La TB y DM constituyen un problema de salud pública de gran importancia, ya que se presentan en forma conjunta y requieren un abordaje, evaluación y manejo integral. Debido a esto, en octubre de 2018 inició la estrategia de atención integral de pacientes con TB y DM en seis unidades comunitarias de salud familiar (UCSF) de la región metropolitana, seleccionadas por tener mayor afluencia de pacientes y por contar con especialistas (UCSF-Especializada San Martin, Barrios y San Jacinto, UCSF-Intermedia Apopa, San Miguelito y Concepción). Esta estrategia incluye la búsqueda y tamizaje a través de la glucometría en personas sintomáticas respiratorias con riesgo de padecer DM, esto con el objetivo de detectar de forma temprana y oportuna la comorbilidad y brindar una atención integral.

Este estudio tiene como objetivo establecer la asociación entre la TB y la DM en establecimientos donde se implementa el plan de atención integral TB-DM.

\section{Metodología}

Este estudio es transversal analítico donde se estudió el total de casos nuevos de TB en todas las formas con y sin DM, mayores de 10 años, que no presentaron otro factor de riesgo (coinfección TB-VIH, enfermedad renal crónica, enfermedades que provocan inmunodeficiencias y personas privadas de libertad).

La recolección de casos de TB y TB-DM se realizó en el periodo de enero a junio del 2019 a partir del registro general de casos de tuberculosis (PCT-5) de la región de salud metropolitana, que es alimentado por cada
UCSF que implementa el plan de atención integral TB-DM. En el periodo seleccionado se registraron 72 casos nuevos de TB, de los cuales se excluyeron 21 (ocho con VIH, cinco menores de 10 años, tres privados de libertad, tres pacientes con pérdida de seguimiento, uno con enfermedad renal crónica y un caso de recaída) quedando un total de 51 casos.

Para la tabulación y elaboración de la base de datos se utilizó Excel. En primer lugar, se analizó la normalidad de los datos cuantitativos a través de la prueba ShapiroFrancia. Se realizó un análisis de medidas de tendencia central y se calculó la prevalencia por sexo y total por cada establecimiento de salud y a nivel general. Se calculó la diferencia entre la prevalencia de DM en los casos de TB y la prevalencia de DM en la población general, también se calculó la razón de prevalencia (RP) para establecer la asociación de padecer TB entre los diabéticos en comparación con los no diabéticos. Para el cálculo de la RP se obtuvo la población mayor de 10 años con DM que consultaron por primera vez y subsecuentemente a través del sistema de morbimortalidad en la web del Ministerio de Salud. Los análisis estadísticos fueron realizados con el paquete estadístico EPIDAT 4.2.

Este estudio es de riesgo mínimo debido a que no se realizaron intervenciones directas con individuos y cuenta con la aprobación del comité de ética en investigación de la región de salud metropolitana.

\section{Resultados}

De los 51 casos nuevos de TB de todas las formas, 57\% (29) eran mujeres. La edad promedio fue de 44 años $( \pm 17.9$ ) y casi la mitad (49\%) procedía de San Salvador y el 31\% (16) de Apopa. La prevalencia de DM en pacientes con TB para los establecimientos que implementan el plan de atención integral TB/DM fue de $23.5 \%$ (IC $95 \% 12.8$ - 37.5), con una prevalencia en mujeres de $24.1 \%$ (IC $95 \% 10.3-43.5$ ) y en hombres $22.7 \%$ (IC $95 \% 7.8$ - 45.4). La prevalencia de UCSF Apopa fue de $18.8 \%$ (IC $95 \% 4.0$ - 45.6); UCSF Barrios $14.3 \%$ (IC $95 \% 0.3-57.8$ ); UCSF Concepción $37.5 \%$ (IC $95 \% 8.5$ - 75.5); UCSF San Jacinto $20.0 \%$ (IC $95 \% 0.5$ - 71.6); UCSF San Miguelito $40.0 \%$ (IC $95 \% 12.2$ - 73.8) y UCSF San Martin 0 (Tabla 1).

La prevalencia de DM en las personas con TB registradas en los establecimientos donde se implementa el plan de atención integral TB-DM, es mayor que la prevalencia a nivel nacional $(p<0.05)$. Sin embargo, no existe diferencia significativa entre la prevalencia de DM identificada en hombres y 
Tabla 1. Prevalencia de DM-TB por sexo según los establecimientos del plan de atención integral TB-DM

\begin{tabular}{lrrrr}
\hline Establecimiento & \multicolumn{2}{c}{ Prevalencia por sexo } & Prevalencia \\
& $\begin{array}{c}\text { Femenino } \\
\mathrm{n}=7\end{array}$ & $\begin{array}{c}\text { Masculino } \\
\mathrm{n}=5\end{array}$ & IC (95\%) \\
\hline UCSF Apopa & $20 \%$ & $16.7 \%$ & $18.8 \%$ & $4.0-45.6$ \\
UCSF Barrios & $20 \%$ & $0 \%$ & $14.3 \%$ & $0.3-57.8$ \\
UCSF Concepción & $50 \%$ & $0 \%$ & $37.5 \%$ & $8.5-75.5$ \\
UCSF San Jacinto & $0 \%$ & $33.3 \%$ & $20 \%$ & $0.5-71.6$ \\
UCSF San Miguelito & $25 \%$ & $50 \%$ & $40 \%$ & $12.2-73.8$ \\
UCSF San Martin & $0 \%$ & $0 \%$ & $0 \%$ & $0-0$ \\
Plan Piloto TB/DM & $24.1 \%$ & $22.7 \%$ & $23.5 \%$ & $12.8-37.5$ \\
\hline
\end{tabular}

Tabla 2. Prevalencia de DM-TB en los establecimientos del plan de atención integral TB-DM y a nivel nacional

\begin{tabular}{lccc}
\hline Sexo & $\begin{array}{c}\text { Prevalencia DM/TB Plan atención } \\
\text { integral \% } \\
\mathrm{n}=12\end{array}$ & $\begin{array}{c}\text { Prevalencia DM según ENECA \% } \\
\mathrm{n}=487875\end{array}$ & Valor de $\mathrm{p}$ \\
\hline Femenino & 24.1 & 13.9 & 0.09 \\
Masculino & 22.7 & 10.6 & 0.07 \\
Plan Piloto TB/DM & 23.5 & 12.5 & $<0.05$ \\
\hline
\end{tabular}

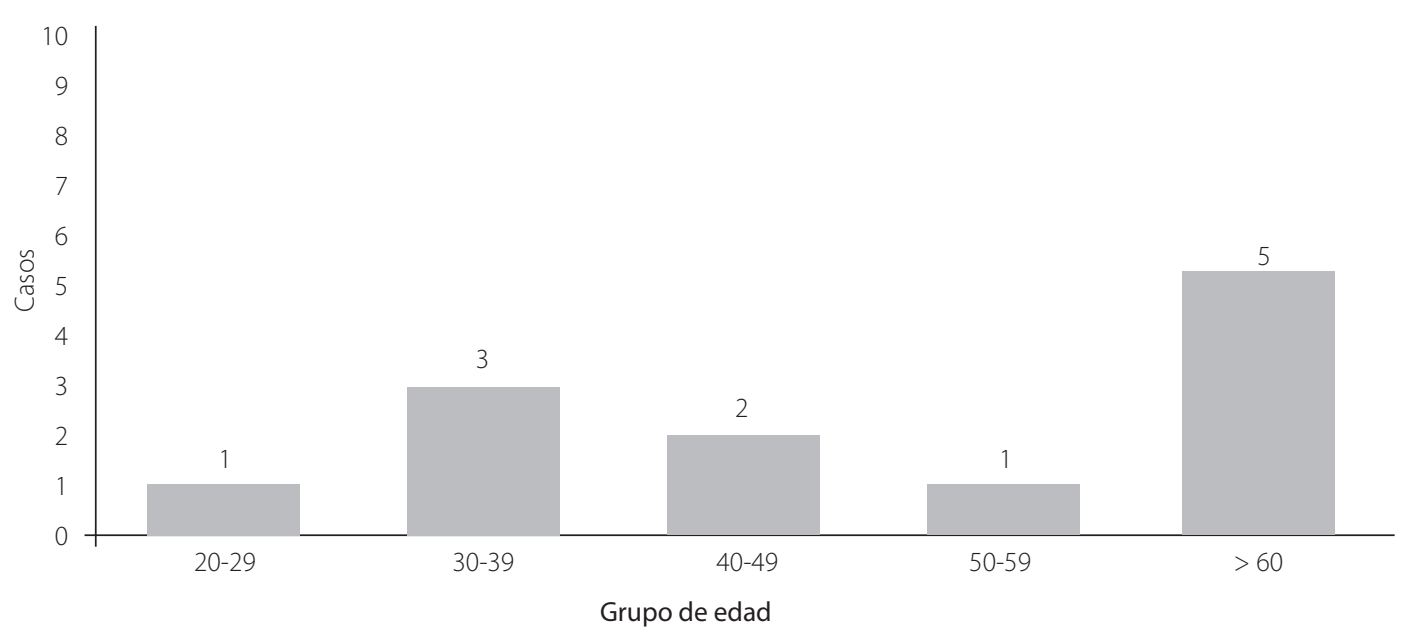

Figura 1. Distribución de las personas con TB-DM por grupo de edad

mujeres registrados en los establecimientos que implementaron el plan y la identificada en la encuesta nacional de enfermedades crónicas no transmisibles en población adulta de El Salvador (Tabla 2).

Solamente a 12 personas se les detectó la comorbilidad TB-DM, de estos, siete eran del sexo femenino. La media de edad de los casos TB-DM es de 51 años $( \pm 16.5)$. Se presentó un caso en el grupo de edad de 20 - 29 años, tres en el grupo de edad entre 30 - 39 años, dos en el grupo de 40 - 49 años, uno en el grupo de 50 - 59 años y cinco en personas mayores de 60 años (Figura 1). No se identificaron casos en el grupo de edad de 10 - 19 años.
De los casos de TB-DM siete procedían del municipio de San Salvador, tres de Apopa, uno de Soyapango y uno de Colón. Once de estos fueron de TB pulmonar y uno TB extrapulmonar. Los 12 casos TB-DM fueron diagnosticados a través de BK, siete con GenXpert y uno a través de cultivo (Figura 2).

Existe una asociación significativa entre la DM y la TB. La TB es 11.4 veces más frecuente en diabéticos que en no diabéticos (Tabla 3).

\section{Discusión}

La asociación entre TB y DM ha sido poco estudiada en El Salvador. En los establecimientos en estudio la probabilidad de padecer TB 


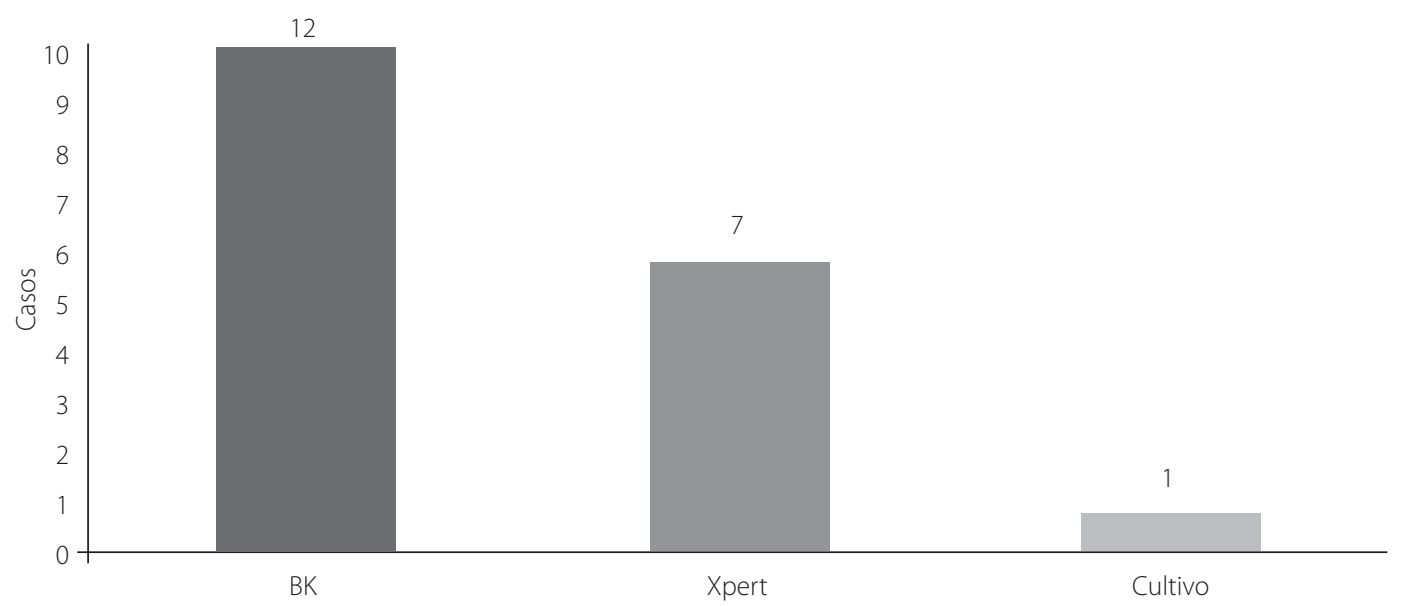

Figura 2. Método diagnostico utilizado para la detección de la tuberculosis

Tabla 3. Asociación de diabetes mellitus y tuberculosis entre personas diabéticas y no diabéticas de los establecimientos que implementan el Plan de atención integral de tuberculosis y diabetes mellitus

\begin{tabular}{lclll}
\hline $\begin{array}{l}\text { Plan atención } \\
\text { integral TB/DM }\end{array}$ & Número de casos & $\begin{array}{l}\text { Prevalencia de } \\
\text { exposición }\end{array}$ & $\begin{array}{l}\text { Razón de } \\
\text { Prevalencia }\end{array}$ & IC \\
\hline TB en diabéticos & 12 & 0.23 & 11.4 & \\
& & & & \\
TB en no diabéticos & 39 & 0.02 & \\
\hline
\end{tabular}

en los diabéticos fue mayor que en los no diabéticos, comparado con otros estudios realizados en Chile y México5. Un estudio que analiza esta problemática a nivel mundial reporta un valor de asociación máximo de $8.3^{6}$, el cual es muy inferior al reportado por este estudio.

Más de la mitad de los casos nuevos de TB-DM eran del sexo femenino. Estos datos son diferentes a los presentados en un estudio realizado en la región metropolitana de Chile ${ }^{7}$, en el cual se evidencia que la mayor parte de casos se presentan en hombres. Sin embargo, la prevalencia de DM-TB entre mujeres y hombres no es estadísticamente significativa, no como en otros estudios realizados en Chile, donde la prevalencia es mayor en mujeres?

La localización de la TB fue mayor a nivel pulmonar, por arriba del porcentaje observado en otros estudios ${ }^{4,7}$. Solo a unos pocos casos se les indicó cultivo y prueba molecular (GenXpert) según lo establecido por la norma técnica para la prevención y control de la TB' ${ }^{8}$. Casi en la mitad de los casos no se cumplió con la normativa y se diagnosticó con baciloscopía.

Los casos TB-DM detectados provenían principalmente de municipios con una gran densidad poblacional, tales como San Salvador, Apopa, Soyapango y Lourdes Colon, que además coincide con la prevalencia más elevada de DM a nivel del país. Como es la región metropolitana del departamento de San Salvador ${ }^{9}$, área urbana que al igual que en un estudio realizado en la India en el año $2007^{10}$, se encontró que la mayor prevalencia de TB se daba en el área urbana que en la rural. Esto no se pudo comprobar en el presente estudio, ya que los establecimientos del plan piloto solo atienden población del área urbana.

La prevalencia en los establecimientos que implementan el plan de atención integral para la TB-DM es similar a la reportada en México ${ }^{11}$, país que presenta condiciones socioeconómicas y culturales parecidas a las de El Salvador y mayor a la prevalencia reportada en las Américas ${ }^{12}$.

A pesar de que todas las UCSF atienden población con características similares, solo se podría atribuir la mayor detección de casos TB-DM a una mayor sensibilidad y mejor organización por parte del personal de salud. Cabe mencionar que el tamaño de la muestra depende de la búsqueda y detección de casos de cada UCSF en estudio, lo que se constituyó en una limitante, ya que no permite inferir los resultados para la población del país, solamente en los 6 establecimientos donde se ha aplicado el plan. Por tal motivo se considera importante implementar a nivel nacional la estrategia de atención integral TB-DM, con el objetivo de 
estandarizar la búsqueda de comorbilidad TB-DM desde la detección del sintomático respiratorio. Así mismo, es necesario la ejecución de estudios a nivel nacional que permitan conocer la prevalencia de TB-DM en el país.

\section{Conclusiones}

La tuberculosis y la diabetes mellitus están asociadas, con mayor probabilidad de padecer tuberculosis en las personas con diabetes que en las que no tienen esta enfermedad. La prevalencia de diabetes es mayor en las personas con tuberculosis que en la población general. La comorbilidad TB-DM afecta más a las mujeres adultas del municipio de San Salvador, quienes en su mayoría presentan una tuberculosis a nivel pulmonar.

\section{Agradecimientos}

Se agradece a los equipos de TB-DM de los niveles locales, del Programa Nacional de Tuberculosis y Enfermedades Respiratorias, de la región de salud metropolitana y nivel central, por el apoyo para el estudio realizado.

\section{Conflicto de intereses}

El equipo de investigación expresa no tener ningún tipo de conflicto de interés. El financiamiento fue por fuentes propias.

\section{Referencias Bibliográficas}

1. Organización Mundial de la Salud. Primera conferencia ministerial mundial de la OMS poner fin a la tuberculosis en la era del desarrollo sostenible: una respuesta multisectorial [Internet]. Moscu: OMS; 2017 nov [citado 25 de octubre de 2019]. Disponible en: $\underline{\text { https://bit.ly/37tSx3e }}$

2. Ministerio de Salud de El Salvador, MINSAL. Programa Nacional de Tuberculosis y Enfermedades Respiratorias, PNTYER. Informe anual de la situación epidemiologica de la tuberculosis en El Salvador 2016 [Internet]. MINSAL. [citado 6 de septiembre de 2019]. Disponible en: https://bit.ly/2ubm2lQ

3. Sistema Único de Información en Salud, SUIS. Reporte situación epidemiologica de enfermedades metabolicas y la nutrición, El
Salvador, semana epidemiologica 1-52, año 2016 [Internet]. [citado 6 de septiembre de 2019]. Disponible en: https://bit.ly/35nlQ6h

4. Ramonda CP, Pino ZP, Valenzuela C LI.

Diabetes mellitus como factor predictor de tuberculosis en el Servicio de Salud Metropolitano Sur en Santiago, Chile. Revista chilena de enfermedades respiratorias. 2012; 28(4):277-85. DOI: https://doi.org/10.4067/ s0717-734820120004000003

5. Ponce-de-León A, García-García ML, García-Sancho MC, Gómez-Pérez FJ, Valdezpino-Gómez JL, Olaiz-Fernández G, et al. Tuberculosis and Diabetes in Southern Mexico. Diabetes Care. 2004; 27(7). DOI: https://doi.org/10.2337/diacare.27.7.1584

6. Dooley KE, Chaisson RE. Tuberculosis and diabetes mellitus: convergence of two epidemics. Lancet Infect Dis. Diciembre de 2009; 9(12):737-46. DOI: https://doi. org/10.1016/s1473-3099(09)70282-8

7. Herrera MT, Leiva ZE, Martín HF, Miranda OM, Morales OC. Asociación entre tuberculosis y diabetes mellitus en la región metropolitana. Rev chil enferm respir. 2013; 29(3):171-5. DOl: https://doi.org/10.4067/ $\underline{\text { s0717-73482013000300008 }}$

8. Programa Nacional de Tuberculosis y Enfermedades Respiratorias. Norma técnica para la prevención y control de la tuberculosis [Internet]. El Salvador: MINSAL; 2018 [citado 20 de septiembre de 2019]. Disponible en: https://bit.ly/2sBqiRl

9. Instituto Nacional de Salud de El Salvador. Resultados relevantes encuesta nacional de enfermedades crónicas no transmisibles en población adulta de el Salvador (ENECA-ELS 2015) [Internet]. [citado 4 de octubre de 2019]. Disponible en: https://bit.ly/2Fh4GN1

10. Stevenson CR, Forouhi NG, Roglic G, Williams BG, Lauer JA, Dye C, et al. Diabetes and tuberculosis: the impact of the diabetes epidemic on tuberculosis incidence. BMC Public Health. 2007; 7(1):234. DOl: https:// doi.org/10.1186/1471-2458-7-234

11. Dávila Y, Castellanos M, García M. Advancement of the national strategy for integrated care in Tuberculosis and Diabetes Mellitus in Mexico, 2015. International Journal of Integrated Care (IJIC). 2015;15:1169. DOI: https://doi.org/10.5334/ijic.2338

12. Barceló A, del Granado M, Castellanos LG, Cotelea S. La amenaza dual de la diabetes y la tuberculosis en las Américas [Internet]. [citado 28 de agosto de 2019]. Disponible en: https://bit.ly/2RwMa9d 